\title{
Afrontamiento generativo de crisis y conflictos en organizaciones*
}

\author{
Dora Fried Schnitman \\ Fundación Interfas, Buenos Aires
}

Recibido: 17 de febrero del 2011 / Aprobado: 15 de marzo del 2011

Este artículo entiende el análisis y la gestión generativa de conflictos y crisis como un nuevo paradigma en comunicación, y como una transformación cultural orientada a promover un abordaje productivo. Para ello presenta las nociones de conflicto, crisis y afrontamiento, los diferentes tipos de conflictos y crisis, las etapas de un proceso de crisis, las respuestas de las personas a los conflictos y crisis. Entiende el afrontamiento como una forma de coordinación social que requiere disposición a trabajar con otro(s). Implica que las personas puedan vincularse entre sí, escuchar lo que cada uno puede aportar, clarificar contextos y propósitos, poner el foco en los temas a resolver, expresarlos de manera adecuada y construir posibilidades que promuevan recursos para acciones especificas.

afrontamiento / generativo / tipos de conflicto / crisis

\section{A generative approach to facing crisis and conflicts in organizations}

This article understands the analysis and generative management of crises and conflict as a new paradigm in communication, and as a component of a cultural transformation geared towards furthering a productive approach thereto. To that end, it presents the notions of conflict, crisis and generative confrontation; different sorts of conflict and crisis; the phases of a crisis process, and the responses of persons to conflicts and crises. Generative confrontation in understood as a form of social coordination that equires willingness to work with others. It entails individuals making connections, listening to what others have to contribute, clarifying contexts and aims, focusing on the issues to be resolved and expressing those issues appropriately, and constructing possibilities that promote resources for specific actions.

generative / confrontation / conflict types /crisis

* Trabajo elaborado a partir de Fried Schnitman, D. (en prensa). Afrontamiento generativo de crisis y conflictos. En Fernández, I. (Comp.). Innovaciones en psicología de las organizaciones. Buenos Aires: J. C. Sáez Editor. Agradezco a Ignacio Fernández, Jorge Sanhueza, Jaime Acuña y Pablo A. Fuenzalida, por su apoyo en la realización de este trabajo.

Correo electrónico: dschnitman@fibertel.com.ar

Persona 14, enero-diciembre del 2011, ISSN 1560-6139, pp. 11-40 


\section{INTRODUCCIÓN}

Cuando hablamos de conflicto nos referimos a diferencias entre personas que pueden resolverse de maneras productivas, estancadas, polarizadas o parciales. La noción de crisis remite a cambios de diversa intensidad, bruscos o crónicos, que producen un desajuste en el funcionamiento de una organización, y entre esta y el contexto; la crisis puede transformarse, cronificarse o intensificarse.

Las situaciones de conflicto y crisis son momentos dolorosos y, a menudo, las personas confrontan entre sí en lugar de afrontarlos. La confrontación tiene una cualidad que se opone al manejo o la resolución efectiva, es decir, al afrontamiento.

El afrontamiento remite a la acepción de la palabra crisis como oportunidad, elección de un camino, transformación y construcción de un futuro. Del latín affrontare, de frons, frontis, frente: poner cara a cara los recursos, los problemas y las posibilidades; hacer frente a un desafío, peligro, crisis, problema o situación incierta o comprometida (Fried Schnitman, 2005, 2008b).

Cuando las personas se encuentran en situaciones de crisis o conflictos con frecuencia experimentan desorientación y turbulencias afectivas, y responden con desencuentros serios, intentos parciales o inestables de reiterar soluciones exitosas en el pasado pero no adecuadas en el presente. Son instancias que mo- vilizan fuertes emociones de desafío, estrés, estupor, dolor, desconcierto en quienes están involucrados y necesitan poder manejarlas en búsqueda de alternativas viables. Las situaciones de conflicto y crisis se caracterizan por una disminución del consenso y la habilidad de las personas para actuar coordinadamente. El impacto de los conflictos y las crisis opaca el sentido de identidad, cuestiona la validez de las relaciones y resquebraja la trama social llegando a veces a su ruptura. Las personas involucradas necesitan construir nuevos sentidos, relaciones y acciones para afrontar la crisis (Fried Schnitman, 2000a).

Los conflictos y las crisis son endémicos a las organizaciones, lo importante es cómo se gestionan en los niveles personal y organizacional. Algunos son acotados, otros necesitan una reorganización más amplia de la estructura y la dinámica organizacional. Algunos pueden ser agudos, otros crónicos.

Los conflictos y las crisis afectan la calidad de la vida en las organizaciones, ponen a prueba su capacidad de afrontar los desafíos y requieren habilidades de estas y de las personas para avanzar más allá de los recursos con los que contaron hasta ese momento. Cuando la dinámica de estos procesos escala puede conducir a las personas y organizaciones a conflictos inexorables o crisis a veces terminales; pero también pueden transformarse y encontrar nuevas formas de adecuarse al problema y al contexto. 
Según Drucker (1999), los miembros de las organizaciones y aun los profesionales no son conscientes de los supuestos básicos con que operan en los procesos de gestión. Sin embargo, estos supuestos -denominados paradigmas en las ciencias sociales- determinan aquello que en la organización se asume como "la realidad" y los procedimientos a seguir, incluyendo los sistemas explicativos de las situaciones de crisis o conflicto y los recursos con que se abordan. En estas circunstancias, dichos paradigmas frecuentemente deben ser reformulados.

Berger y Luckmann (1966) han planteado que virtualmente toda la experiencia se organiza, interpreta y comprende mediante sistemas explicativos que las personas desarrollan en sus interacciones; estos sistemas operan como intermediarios entre ellos y los contextos. Los sistemas explicativos son producto de acciones sociales previas, presentes o futuras, y el sentido de objetividad que adquieren resulta del consenso y de compartirlos con otros. Cuando son compartidos, proveen de principios que ligan las acciones conjuntas, el devenir de los hechos, las narraciones y significados del mundo perceptual. También proveen a las personas del sentido acerca de qué lugar ocupan en el grupo. Estos conjuntos o sistemas de relaciones explicativas permiten distinguir lo significativo para cada uno y para el conjunto, dan lugar a comprensiones, acciones, expectativas, y a la habilidad de predecir y controlar. Así, los miembros de un grupo desarrollan en su interacción nociones compartidas sobre diferentes dimensiones de sí mismos, su accionar y su mundo social.

Entendiendo la comunicación como coordinación de acciones entre actores sociales -no solo como transmisión de información-, en las últimas décadas las organizaciones se consideran y abordan como redes de conversaciones que coordinan acciones con distinto grado de eficacia según el momento y el contexto. Estas redes se constituyen a través de co-construcciones y acciones conjuntas que tienen lugar en los procesos de gestión. Los supuestos, los sistemas explicativos y las conversaciones articulan el funcionamiento de las organizaciones. Las situaciones de conflicto y crisis quiebran dicha articulación.

Para establecer conversaciones productivas que restituyan el funcionamiento o lo modifiquen, los miembros de una organización necesitan flexibilizar o transformar los sistemas explicativos y los supuestos con los que operan, admitir otras perspectivas, adecuar las acciones e interacciones a las situaciones a resolver y considerar un rango más amplio de alternativas. Estos procesos tienen lugar en conversaciones que pueden flexibilizar los sistemas explicativos y las acciones conjuntas, incluyendo las opciones inéditas.

La capacidad de afrontamiento alude a la recuperación o desarrollo de competencias y recursos para promover 
conversaciones productivas, coordinaciones sociales y diseño de los procedimientos necesarios para desplazarse hacia alternativas y nuevas posibilidades. El afrontamiento implica aprendizaje reflexivo, innovación, foco y creatividad social. El diálogo es el medio y el instrumento de este proceso.

Las crisis y los conflictos pueden incluir alternativas $\mathrm{u}$ opciones que permitan el crecimiento de las personas y organizaciones: cuando se afrontan con la manera y los tiempos adecuados; cuando se dan las condiciones para una reconsideración de los sistemas explicativos personales y organizacionales; cuando la comunicación deviene productiva y genera acciones viables; cuando las emociones pueden ser reorientadas positivamente; cuando los aportes recíprocos permiten avanzar posibilidades, captar oportunidades, reciclar las habilidades y competencias; cuando pueden aprender. En síntesis,

Denominamos afrontamiento de crisis y conflictos al diseño y la implementación de procedimientos necesarios para abordar adecuadamente las situaciones específicas de conflicto y crisis, con la aspiración de que resulten efectivos. Abarca la elaboración de supuestos y sistemas explicativos alternativos, la construcción de conversaciones productivas, la colaboración y la coordinación entre los involucrados, con un foco en la resolución del conflicto o crisis en curso. cuando las personas pueden afrontar en lugar de confrontar.

PROFUNDIZACIÓN DE LA CRISIS VS. AFRONTAMIENTO

Más allá de su origen, las crisis se profundizan o se procesan por la interacción que se establece entre factores crisógenos, contextos y respuestas de los participantes.

La interacción entre los factores crisógenos, la gestión y los contextos van marcando el rumbo del proceso. Si los procesos de crisis se intensifican se desarticula progresivamente la posibilidad de funcionamiento de la organización. En la interacción social está implícito qué es lo adecuado, qué corresponde, qué se espera de los otros.

No hay crisis per se, hay procesos de crisis y gestiones de crisis.

Cuando las crisis o los conflictos no se abordan en tiempo y forma el impacto y la desorganización avanzan limitando progresivamente las competencias para afrontar los desafíos. Así, se verán limitados el registro de los contextos internos y externos y las reformulaciones operativas acerca de la crisis, las competencias para realizar transformaciones, la capacidad de acción, la conectividad entre personas, la capacidad para participar en diálogos productivos, el registro de las emocio- 
nes y el sentido de identidad de unos y otros.

Si los conflictos y las crisis se profundizan los procesos pueden conducir a la anomia, la polarización, la atribución de culpas, el foco en un aspecto parcial o la imposibilidad de discriminar los temas importantes y ordenar su resolución en el tiempo. Los supuestos y sistemas explicativos que se utilizaban y las prácticas consecuentes pierden la eficacia, la congruencia y la posibilidad de generar respuestas adecuadas. Los miembros de una organización encuentran que su experiencia subjetiva y las acciones que podrían llevar adelante ya no calzan adecuadamente con esta ni con el contexto.

Una de las manifestaciones de una crisis es el resquebrajamiento de la dinámica organizacional y la necesidad de adecuación a las nuevas circunstancias que, sin embargo, no pueden materializarse. Se instalan la desazón, la desesperanza, la desorientación; disminuye la capacidad de emprender y cada persona está cada vez más aislada y con menos posibilidades de coordinar con otros. A menudo las crisis provocan confusión o turbulencia y no se encuentran fácilmente las respuestas, produciéndose demoras a veces inadecuadas.

Las situaciones de crisis y su afrontamiento incluyen procesos y tiempos muy diversos que requieren diferentes tipos de acciones: reflexión, ponderación del contexto y los recursos, encon- trar recursos para articular un cambio, vincular posibles acciones a contextos específicos, intercambiar acerca de las ventajas y desventajas de los cursos posibles, ajustar el foco y los propósitos de la acción, actuar, evaluar, aprender y avanzar hacia un manejo de la situación.

En los procesos de afrontamiento es necesario que los participantes puedan considerar la situación y el contexto en cuestión, crear condiciones necesarias para seleccionar los objetivos, priorizar temas, coordinar rumbos, reconocer las emociones positivas y negativas, apoyarse en los vínculos existentes o posibles, o reformularlos, y restituir las condiciones de seguridad y confianza cuando resulte adecuado promover conversaciones productivas.

Esto no sucede cuando:

- Disminuyen el consenso y la habilidad para actuar coordinadamente.

- Se opaca el sentido de identidad y las expectativas recíprocas.

- Se cuestiona la validez de las relaciones.

- Se genera aislamiento.

- Se genera inculpación recíproca.

- Se incrementan la rigidez y las demandas de un funcionamiento que ya no es necesariamente válido.

- Las personas pierden la capacidad de tomar responsabilidad.

- Se disuelve la trama social y a veces se llega a una crisis profunda o a una ruptura. 


\section{CARACTERIZACIÓN DE CONFLICTOS Y CRISIS}

Origen y duración de la crisis

- En las crisis de origen externo las situaciones traumáticas y estresantes impactan sobre las personas y organizaciones, y desafían la viabilidad de sus recursos habituales para responder. Las crisis de origen interno son resultado de situaciones internas a la organización.

- Las situaciones de crisis aguda o abrupta requieren acciones inmediatas, las decisiones deben tomarse muy rápido por la complejidad y la urgencia. Es preciso actuar, establecer un foco claro y movilizar los recursos necesarios en lo inmediato; sin embargo, en un momento posterior, cuando se haya dado respuesta a la emergencia, será necesario considerar las situaciones que quedaron fuera del foco de la acción.

- Las crisis crónicas requieren un esfuerzo sostenido y una solución adecuada a su naturaleza prolongada. Es importante permanecer alertas al desgaste y los conflictos que surjan por el esfuerzo sostenido, discernir las acciones que se toman jerarquizando lo significativo en los diferentes momentos para administrar los recursos.

\section{Grado y profundización de conflictos y crisis}

A continuación incluimos una breve descripción de los diferentes tipos de conflictos y crisis. Es importante que el profesional y los participantes los identifiquen porque requieren habilidades específicas para afrontarlos. Unas veces corresponden a etapas progresivas de una misma crisis, otras a distintos estados según su grado de profundización. En todas las circunstancias es preciso atender tanto a la calidad de las relaciones como al contexto (Fried Schnitman, 2005; Pearce \& Littlejohn, 1997).

- Tipo A.- Los participantes todavía comparten una cultura organizacional, una interpretación del contexto, reconocen y responden -aunque sea parcialmente- a valores, visiones y a un conjunto de reglas compartidas, conservan un sentido de pertenencia aunque la trama social esté afectada. En estas situaciones, si bien existen conflictos y crisis los sistemas explicativos, las maneras de comprender y explicar la realidad y la visión acerca de las circunstancias en juego aún son parcialmente compatibles. El afrontamiento se puede facilitar incrementando las habilidades para sostener conversaciones productivas -las que fluyen hacia coordinaciones sociales y organizacionales posibles- y flexibilizar los sistemas explicativos en las áreas que resulte necesario abordar para considerar alternativas. Si sostienen una buena comunicación pueden escuchar $\mathrm{y}$ comprender mejor los planteos de los otros, reconocer sus contribuciones para afrontar positivamente, 
construir marcos compartidos, encontrar soluciones creativas y avanzar aprendiendo en el curso de la experiencia.

- Tipo B.- Los sistemas explicativos de los participantes se vuelven muy diferentes y en algunas circunstancias incompatibles, afectando la coordinación de acciones comunes, desdibujando la trama social -el nosotros-, la posibilidad de establecer acuerdos, el sentimiento de pertenencia y la posibilidad de sostener conversaciones en las que puedan comprenderse recíprocamente. Bernstein (1985) utiliza el concepto inconmensurable para describir la relación entre perspectivas o realidades sociales que difieren de manera tal que una no puede ser expresada o comprendida dentro de la otra. En la medida en que es imposible incorporar la perspectiva del otro en la propia habitualmente se generan malestar, rigidez y procesos de inculpación. En estas circunstancias es difícil afrontar conflictos o situaciones crisógenas sin una transformación de los sistemas explicativos y operativos de los participantes.

Es preciso encontrar un marco común para construir nuevos sistemas explicativos y operativos: se trata de desarrollar una nueva matriz generativa de significados, visiones $y$ acciones que facilite el afrontamiento, de promover coordinaciones y acciones en dirección a aquello que se necesita resolver.

Esta matriz permite transformar la manera de comprender e interpretar los acontecimientos, construir significados comunes, mejorar la calidad de las conversaciones, considerar las situaciones desde otras ópticas que posibiliten la implementación y el sostén de acciones conjuntas para manejar la crisis. Puede ser acotada a aquello que es necesario coordinar para sostener acciones de afrontamiento o extenderse a aspectos más amplios de la organización. Esto permite encontrar nuevas perspectivas, significados y argumentos compartidos, desarrollar nuevos cursos de acción, y recuperar "el nosotros" y las emociones positivas. En los ejemplos se observa la construcción de una matriz generativa para la gestión personal y organizacional.

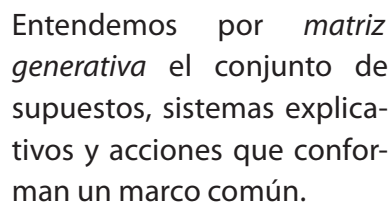
generativa el conjunto de supuestos, sistemas explicativos y acciones que conforman un marco común.

- Tipo C. Los sistemas explicativos se han tornado incompatibles. Se asume que el otro y lo significativo para él no son importantes o son imposibles de compatibilizar con lo significativo para uno. La trama social se ha roto, se ha perdido la confianza, las personas y los vínculos ya no son conside- 
rados y no se sostiene el sentido de lo común. La organización pierde su integridad como tal y su capacidad de respuesta, no hay acciones coordinadas, cada persona o sector se focaliza en su propia supervivencia. Habitualmente, la estrategia de las personas o sectores consiste en tratar de imponer la propia perspectiva del mundo sobre la del otro, a pesar de los mejores esfuerzos que el otro pueda hacer para evitarlo. En este caso, se tratará de construir condiciones para recuperar la noción de lo común "al menos en el área involucrada por la crisis": lo común que permita estar al timón y resolver los temas en cuestión. Este tipo de conflictos y crisis requieren necesariamente la intervención de profesionales porque los recursos de la organización se agotaron en acciones fragmentadas. Si no pueden retrotraerse o resolverse, a menudo conducen al estancamiento y la disolución.

Impacto de la profundización de la crisis

A medida que la crisis se acentúa, el clima de la organización se modifica. Progresivamente se incrementan la inculpación recíproca, el enojo, el resentimiento, la intolerancia, y las discusiones aparecen y desaparecen de acuerdo con patrones azarosos. La desorganización de los patrones y creencias, y la pérdida del consenso llevan a que cada miembro sienta que no sabe quién es -ni como persona ni dentro de la organización-y hasta sienta que su vida se disuelve, que sus vínculos con otros y su pasado se tornan vulnerables y pueden romperse. Vemos así la transición de un tipo de conflicto a otro a medida que la crisis se acentúa. Una de las posibilidades es retrotraer la crisis a condiciones operativas viables, contemplar asociaciones útiles, interna o externamente; o considerar negociaciones productivas para retrotraer la crisis y afrontarla; cuando esto no sucede se puede instalar una crisis crónica o llegar a la disolución de la organización. También se puede reorientar hacia un futuro diferente consensuando una mejor coordinación, que transforme las relaciones y mejore la trama social.

Las personas frente a conflictos y crisis: Gestión del sí mismo, gestión organizacional

En algunas circunstancias los participantes están desorientados, no pueden dirimir el marco o los términos de las contradicciones, y esto les impide formular de manera sistemática los temas problemáticos a considerar y los cursos de acción posibles; les resulta difícil reconocerse a sí mismos como parte activa de la construcción de posibilidades debido a las contradicciones en las que están envueltos. Las contradicciones les parecen inevitables, negativas o inmodificables y ven su mundo social confuso, plagado de dilemas e incertidumbres. Quedan entrampadas en un 
escenario de opciones problemáticas, que reiteran pasiva o activamente $y$, en ocasiones, manifiestan con la ambigüedad de sus respuestas. Para favorecer el afrontamiento el profesional puede orientarse a clarificar marcos y opciones facilitando el reconocimiento de los temas en cuestión, la comprensión de sí, del otro, de los contextos, las diferencias y las posibilidades emergentes.

En otras ocasiones, si bien los participantes toman una posición activa frente al conflicto, no se orientan en el rumbo adecuado: se involucran pero jerarquizan algún aspecto parcial de la contradicción o conflicto, y pueden llegar hasta la negación de aspectos importantes de los temas en cuestión, del otro, de sí mismos o de las circunstancias. También puede suceder que realicen búsquedas forzadas de consenso en aspectos parciales, o insistan en sostener el conflicto incrementando las escaladas y la desorganización, o produzcan respuestas nuevas pero inadecuadas al contexto o al problema. Para favorecer el afrontamiento es necesario que el profesional promueva una comprensión abarcativa de la situación y de las relaciones que no están considerando, y sostenga la posibilidad de diálogo y el reconocimiento de las diferencias; también que se mantenga atento a las posibilidades emergentes que reciclen, transformen o enlacen de maneras novedosas los factores crisógenos y su gestión.

Con frecuencia las personas expresan sus intentos de reorganizarse y su imposibilidad de consolidar las nuevas perspectivas y posibilidades de acción. Pueden aparecer alternativas adecuadas que no logran establecerse y se diluyen. La oscilación resulta algunas veces de la dinámica del proceso mismo; otras, de privilegiar -en determinado momento o contexto- algún aspecto del conflicto. Para favorecer el afrontamiento la estrategia se orienta al reconocimiento y valoración de las innovaciones implementadas y a facilitar su expansión a otras áreas, con un manejo del tiempo que dé lugar a la estabilización de nuevos recursos. Para ello, el profesional puede promover el diálogo con la propia experiencia y el reconocimiento de las posibilidades emergentes. Dichas posibilidades permiten seleccionar operativamente las acciones adecuadas para sostener una opción futura. En otras ocasiones, en cambio, es necesario focalizarse en las condiciones necesarias para expandir el diálogo y apoyar la coordinación entre los participantes.

También es posible construir fórmulas para la acción, improvisaciones pragmáticas que trasciendan las contradicciones o desafíos sin alterar su presencia. Los participantes pueden aceptar y reconocer que las polarizaciones no son reconciliables, pero se reconocen a sí mismos como sujetos-agentes -activos y participantes, con iniciativa para trabajar con otros, dispuestos a integrar grupos-; reconocen, sostienen y asumen la diversidad, y toleran la ten- 
sión; consideran las diferencias y las exploran monitoreando su evolución. Se puede realizar una exploración sistemática de las diferencias y ponderar los recursos para el afrontamiento que ofrece cada uno. Los participantes son capaces de confrontar fuerzas en oposición gestionando pragmáticamente la crisis. Para facilitar el afrontamiento el profesional facilita el reconocimiento de los diversos cursos de resolución posibles, las preferencias de los participantes, la deliberación sobre las ventajas y desventajas de cada uno de ellos, y realiza síntesis parciales reconociendo qué pueden hacer en ese momento los participantes en beneficio del afrontamiento y la búsqueda de un camino viable compartido (Fried Schnitman \& Schnitman, 2000a; 2000b).

\section{Crisis como estado alejado del equilibrio}

En las situaciones de crisis, la dinámica de los grupos humanos empieza a operar en condiciones particulares alejadas del equilibrio: aunque haya un esfuerzo visible por mantener el funcionamiento conocido, la inestabilidad puede conducir a cambios significativos en el grupo.

Podemos asistir al surgimiento de nuevas dinámicas en la organización, cuyas características contrastan marcadamente con las anteriores. En dichas condiciones pueden irrumpir procesos totalmente nuevos o diferentes sin antecedentes en su historia.
En condiciones alejadas del equilibrio, los procesos organizacionales se vuelven particularmente sensibles a las influencias externas y la singularidad de las circunstancias. En los momentos en que se abren diferentes alternativas es imposible predecir o determinar cuál será la dirección del cambio. En el afrontamiento de estas situaciones es importante tolerar las incertidumbres y fluir con las oscilaciones. Sin embargo, también es necesario mantener cierta constancia sin llegar a la rigidez y continuar identificando caminos alternativos posibles.

\section{Resoluciones limitadas de la crisis y estados oscilatorios}

En su resolución de las crisis las organizaciones expresan intentos de reorganizarse e imposibilidad de consolidar los cambios. A veces esta oscilación se convierte en una forma de funcionamiento estable en la que coexisten pautas y premisas previas adecuadas a un contexto que no es el actual con intentos de reorganización. Los estados oscilatorios son frecuentes y en ellos se observan algunas posibilidades operativas novedosas, aunque no pueden establecerse: hay oscilaciones entre el mantenimiento de la organización previa y la búsqueda de una forma nueva. Estos estados oscilatorios se acompañan de discusiones, variaciones en el nivel de tensión, importantes modificaciones afectivas, cambios en la identidad y pérdida de consenso. 
Un segundo tipo de resolución limitada de la crisis será el pasaje a una forma rígida de funcionamiento con consenso forzado, donde se exagera una modalidad previa con menor supervivencia de alternativas.

Un tercer tipo de resolución consiste en un aumento de la desorganización que puede conducir a la disolución.

Finalmente, en otros casos aparecen y persisten respuestas nuevas pero no apropiadas. Las combinaciones de todas estas modalidades de resolución son múltiples. Aunque las alternativas que surjan no lleguen a cobrar suficiente fuerza como para que la organización se reorganice en una modalidad de funcionamiento diferente, es posible encontrarlas si se las observa con una óptica dual de estabilidad y transformación en qué se mantiene igual a sí misma y en qué ha variado; qué pueden reconocer como novedoso, inédito o diferente.

Para favorecer el afrontamiento se reconocerán los cambios y se explorarán las alternativas actuales apreciando los intentos realizados, se identificará lo novedoso y si es adecuado se invitará a implementarlo. Es importante reflexionar sobre cómo sostener los cambios, sus desafíos y aspectos positivos/negativos para clarificar los dilemas que puedan presentarse, y revisar qué podría tornar difícil mantenerlos.

\section{Reorganización exitosa}

Las organizaciones y sus miembros pueden reordenarse si encuentran una manera compartida de afrontar los eventos críticos. La respuesta a la crisis y el tipo de solución requerida se convierte en una alternativa para la reorganización. Estos afrontamientos son construcciones que emergen como respuestas activas en situaciones de crisis, están ligados a los propios esfuerzos de la organización y sus miembros para restaurar su integridad, y pueden promover núcleos activos para el cambio.

Cuando se encuentra una alternativa es necesario expandirla y consolidarla. Este cambio no se establece en un solo movimiento sino que hay dinámicas que unas veces tienden a la desorganización, otras a recrear patrones previos y otras hacia una nueva organización. En este caso resulta importante poder sostener el foco.

Cuando el afrontamiento es adecuado la intervención consistirá en reconocer las competencias, los logros, la participación y las posibilidades de afrontamiento que abren.

\section{Condiciones PARA EL AFRONTAMIENTO}

Para el desarrollo de una adecuada respuesta de afrontamiento se requiere un análisis de los contextos, los valores, las relaciones y los recursos, y una ponderación de los costos y beneficios -tangibles e intangibles- de ajustar, transformar, sostener o desarmar las estructuras existentes en la organización.

El afrontamiento requiere diferentes tipos de iniciativas: 
- Creación de un contexto adecuado: análisis de la situación, los desafíos y recursos, seleccionar los propósitos y objetivos, priorizar temas, encontrar rumbos, apoyarse en los vínculos existentes o posibles, o reformularlos, y restituir las condiciones de seguridad y confianza.

- Facilitación de emociones capaces de sostener el proceso: esperanza, convicción de que es posible encontrar una solución, solidaridad y respeto recíproco, sentido de pertenencia, participación proactiva en la búsqueda de soluciones, coraje.

- Creación de matrices generativas que permitan construir nuevas perspectivas, encuadres y significados; vincular posibles acciones a contextos específicos, intercambiar acerca de cursos posibles, ajustar el foco de acción, avanzar, evaluar, aprender.

- Manejo del tiempo: a menudo las crisis provocan confusión o turbulencia, se producen demoras, a veces inadecuadas; se requiere ecuanimidad y sostén de la alternativa considerada en el tiempo ponderando permanentemente los resultados.

- Creación de relaciones interpersonales de confianza y conectividad: compromiso de coordinación con otros, aceptación de la diversidad, la flexibilización de los sistemas explicativos y las conversaciones con una disposición a escucharse recíprocamente sin perder de vista el objeti- vo de avanzar hacia coordinaciones operativas que permitan afrontar la crisis. Se instala una nueva forma de comunalidad.

- Recuperación y construcción de recursos novedosos: formular síntesis inéditas e inclusivas de las diferentes perspectivas o acciones relevantes, conformar escenarios inesperados para la acción, donde se incorporan la creatividad, la apertura de nuevas potencialidades y el desarrollo de una mirada apreciativa de los aportes recíprocos.

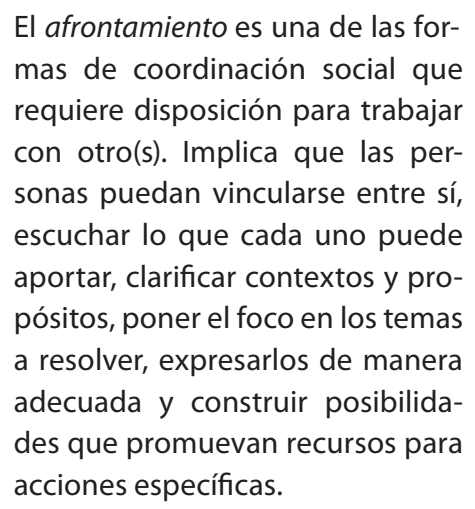

- Gestión del sí mismo: ponderar las contribuciones y recursos que puede aportar cada participante, reflexionar sobre la situación conservando la capacidad de escuchar a los otros participantes y a sí mismo.

- Gestión de la crisis: implica un ajuste permanente de acuerdo a los resultados que se vayan obteniendo, un aprendizaje continuo sobre la 
base de las mejores prácticas y los problemas, y una evaluación de la gestión.

- Productividad de los diálogos e iniciativas: requiere un monitoreo de la evolución del diálogo hacia perspectivas, coordinaciones y acciones que permitan encauzar y avanzar los temas en cuestión.

En un proceso exitoso las personas podrán:

- Trabajar con una lógica de lo posible.

- Encontrar caminos hacia una transformación.

- Construir nuevos significados y posibilidades de acción conjunta.

- Formular argumentos novedosos que trasciendan las perspectivas previas.

- Reconocer oportunidades singulares.

- Promover interpretaciones y acciones novedosas.

- Reconocer interacciones útiles, en algunos casos, inéditas.

- Promover puntos de vista novedosos y lugares sociales legítimos para los participantes.

- Coordinar o negociar encuadres que permitan construir acuerdos y avanzar.

- Identificar o diseñar los cursos de acción que se consideren más productivos, implementarlos $\mathrm{y}$ evaluar su funcionamiento.
- Encontrar un sentido de dirección, saber qué hacer es tan importante como saber cómo hacer -cómo vincular acciones específicas con el contexto y las posibilidades- cuando las personas necesitan centrarse en acciones específicas para relacionar el contexto con las posibilidades. La capacidad de saber expresarse y comprenderse adecuadamente permite dirimir las posibilidades. Saber ubicarse en las diferentes dimensiones de los procesos de conflicto y crisis -saber de sí en contexto-, se acompaña del saber emocional que ofrece un sensor para evitar los trayectos minados y elegir los que ofrecen oportunidades. El saber relacional facilita la construcción de lo común frente a las necesidades, más allá de las diferencias (Fried Schnitman \& Schnitman, 2000a; 2000b).

\section{Comprensión sistémica del contexto} de la crisis

El tránsito desde el reconocimiento de la crisis hasta la situación de afrontamiento es un proceso que es necesario construir paso a paso. Consideraremos algunos aspectos de este proceso:

a) Construir las relaciones necesarias para establecer vínculos de confianza y revisar la información pertinente para conocer las características de la organización y la necesidad de cambio. Entre otros aspectos relevantes, 
esto incluye: tipo y dimensión de la organización y la crisis, composición, organigrama formal, líneas de responsabilidad y poder formales e informales para la toma de decisiones, coordinaciones efectivas entre personas, conformación de los equipos, valores compartidos, calidad de las relaciones, bienes tangibles e intangibles producidos por la organización, un sentido de lo común. El detalle de la información que será necesario recabar dependerá de la singularidad de cada circunstancia; por ejemplo, según el tipo de crisis el estado económico-financiero será esencial pero en otras no. En una crisis medioambiental el estado de situación, recursos humanos y de otra índole necesarios; y así para cada tipo específico de crisis.

b) Contextualizar la crisis en el tiempo aclarando la discontinuidad entre la forma de organización previa, la situación actual y los futuros posibles con el propósito de ubicar la especificidad del momento y el tipo de gestión necesaria. Reconocer diferencias. Comprender los cambios ocurridos. Ubicar la necesidad de cambio en los contextos adecuados.

c) Identificar el tipo y la profundidad de la crisis, y los intentos que se rea- lizaron para gestionarla, exitosos o no. Explorar las relaciones internas en el equipo de crisis y la organización, y las externas con el contexto.

d) Restituir el clima grupal y las competencias necesarias para que las personas puedan buscar opciones, reconocer recursos e innovar. Ampliar la perspectiva, flexibilizar y facilitar el diálogo. Explorar qué contribuciones podrían realizar las personas, equipos, secciones, etcétera. Explorar la disposición para afrontar, establecer acuerdos y compromisos recíprocos.

e) Identificar los caminos posibles para la gestión de la crisis. Explorar las alternativas actuales clarificando los dilemas que presenta el cambio. Buscar situaciones que hayan sido afrontadas exitosamente en el pasado especificando en qué momentos pudieron reorganizarse y en cuáles no, como así también qué favoreció el afrontamiento en ese momento.

f) Ponderar el capital social, relacional, económico y de conocimiento, los valores tangibles e intangibles que se comprometen o preservan en cada instancia, más allá de los aspectos económico-financieros tradicionalmente considerados.

g) Estabilizar los recursos, las alternativas y la participación adecuada. 


\section{Herramientas generativas para la búsqueda de opciones: Preguntas-guía ${ }^{1}$}

En esta sección se incluyen preguntas-guía para formular a los participantes, orientadas a facilitar la emergencia de posibilidades y recursos para el afrontamiento. Estas preguntas expanden las habilidades de las personas para reconocer y utilizar sus propios recursos, favorecer la experimentación, el conocimiento y la comunicación efectiva frente a diferentes requerimientos de un proceso de afrontamiento.

- Preguntas para identificar y describir posibilidades generativas

- ¿Qué nuevas posibilidades tuvieron lugar o podrían considerar frente a los desafíos que están enfrentando? ¿Qué soluciones imaginan?

- ¿Cuáles de ellas podrían contribuir y de qué manera?

- ¿Qué posibilidades de acción ofrecen?

- Si se implementaran esas posibilidades en la situación que enfrentan ¿qué cambios producirían?

- ¿Qué otras posibilidades podrían recuperar o construir?

- ¿Qué futuro podrían construir?

- Preguntas para encuadrar caminos posibles y perspectivas emergentes

- ¿Qué caminos abrirían las posibilidades que están considerando?

- ¿Qué aportarían?

- ¿Cómo podría participar cada uno y el grupo para avanzar?

- ¿Qué despierta su interés? ¿Qué desean o consideran necesario profundizar?

- ¿Cuáles son sus interrogantes, dudas e incertidumbres?

- Preguntas para facilitar el reconocimiento del otro y la constitución de relaciones que promuevan afrontamiento

- ¿Qué escuchó decir al otro?

- ¿Qué aporta?

- ¿Qué nota diferente en las propuestas que permitiría avanzar?

- ¿En qué aspectos se sintió escuchado?

- ¿En qué nuevas situaciones se reconoció involucrado?

- Preguntas para incrementar la solidaridad y la conciencia colaborativa

- ¿De qué manera beneficiarían a la organización y los participantes las nuevas posibilidades que están explorando? ¿De qué manera otro(s) podría(n) contribuir?

- ¿De qué nuevas maneras podrían conversar entre ustedes y con otros para que este proceso resulte productivo?

- ¿Cómo podría involucrarse específicamente cada participante en la construcción de las posibilidades que están considerando? ¿De qué otra forma podría hacerlo él/ella?

1 Otras herramientas generativas se trabajan en otras publicaciones de la autora (2010a, 2010b, 2010c; 2009; 2008a). 


\section{El manejo del tiempo}

El manejo del tiempo y la intensidad con que se deben trabajar las soluciones encontradas son importantes en la gestión de conflictos y crisis. Las nuevas alternativas no se establecen de una vez para siempre ni de una sola movida, sino gradualmente y en áreas limitadas, para expandirse después si cobran suficiente fuerza. Si las alternativas responden adecuadamente a la situación será importante sostenerlas y generar contextos adecuados para su implementación. Es de esperar que haya competencia entre el funcionamiento previo y el sostén de las nuevas alternativas. Desde ese punto de vista es necesario considerar un conjunto de abordajes complementarios que tenderían a cuestionar el mantenimiento de las premisas y acciones vigentes previamente. Por eso es que muchas veces, en la modificación de una organización hace falta promover la interrogación reflexiva, el cuestionamiento, la exploración de las dudas respecto del accionar y las creencias sostenidas previamente, y en ocasiones facilitar su bloqueo activo.

\section{DIÁLOGOS Y MOMENTOS DEL PROCESO}

PARA EL AFRONTAMIENTO

Distinguiremos diferentes tipos de diálogo y momentos en procesos de afrontamiento.

Los diálogos que construyen el contexto y facilitan el consenso se orientan a crear las condiciones que permiten acordar acerca del proceso, su inicio, características, participantes, propósito y desarrollo. Los diálogos generativos se focalizan en la construcción de nuevas posibilidades durante el proceso; proveen de habilidades para generar temas significativos, recuperar recursos, visualizar el futuro, generar involucramiento y acciones participativas. Los diálogos apreciativos recuperan positivamente diferentes aspectos de la vida de las personas, organizaciones, comunidades. Cuando reconocemos los recursos y oportunidades que permiten crear contextos de implementación adecuados utilizamos diálogos posibilitadores. Los diálogos de distinción y organización de diferentes momentos, prioridades, necesidades, contextos, etcétera, permiten ordenar el tiempo y la secuencia de acciones. Los diálogos productivos ponderan si los procesos y relaciones permiten avanzar adecuada y efectivamente en los temas en cuestión. Los diálogos reflexivos remiten a procesos de aprendizaje a partir de la propia experiencia, reconociendo formas novedosas de participación y comprensión que incrementan los recursos. Los diálogos mediadores y negociadores son aquellos que -en medio de diferencias, contradicciones, imposibilidades y conflictos- permiten encontrar síntesis inesperadas en el diálogo tal como tiene lugar; opciones y perspectivas que los participantes pueden asumir como propias, aunque sostengan diferencias.

Es importante que tanto los participantes como el profesional puedan ubicar el momento para avanzar el proceso.

DeL CONFLICTO, LA CRISIS Y EL

AFRONTAMIENTO

Cuando hablamos de conflicto nos referimos a diferencias que pueden ser 
productivas, estancarse o polarizarse. Una crisis remite a la necesidad de un cambio en la organización. Puede ser provocada por cambios bruscos o situaciones crónicas que requieren transformaciones en los procesos organizacionales. Surgen de situaciones internas o del contexto, o bien de situaciones internas que no calzan con los contextos. Las crisis pueden poner en duda la continuidad de la organización y las relaciones, o generar dificultades y desafíos que requerirán una transformación. En una crisis el ajuste entre los supuestos, los sistemas explicativos y las prácticas que se derivan para la organización no son adecuados a la organización y su relación con el contexto. El lugar que las personas ocupan en la organización y las relaciones interpersonales se desarticulan, pierden el sentido. Los cambios críticos no siempre son previsibles y tienen un grado de incertidumbre en cuanto a su resolución, su reversibilidad o grado de profundidad. Las crisis también son coyunturas de cambio, están sujetas a transformaciones. Hemos planteado que las organizaciones operan de acuerdo a sistemas explicativos y de acción, posibilidades que pueden activarse o reciclarse en nuevos contextos, y recursos potenciales para el cambio que junto con el aprendizaje y la capacidad de innovar proveen de la creatividad necesaria en la búsqueda de alternativas. Cuando tienen lugar situaciones de crisis la recuperación de los recursos y la creación de nuevas maneras de comprender y relacionarse permiten la exploración de condiciones para el afrontamiento (Fried Schnitman, 1989; Prigogine, 1979).

El afrontamiento alude a diálogos que permiten operar productivamente en la construcción de nuevos sentidos, realidades y valores utilizando precisamente como medio la conversación y las coordinaciones sociales.

Es importante señalar que en este proceso se genera un espacio social, las formas de participación no surgen de la nada ni tienen un efecto lineal; por el contrario, solo adquieren sentido en el proceso, en la respuesta, la suplementación de sentido de quien escucha o es interlocutor en un diálogo. Quien trabaja generativamente necesita estar atento al espacio social e interpersonal del diálogo, aquello que sucede entre personas y en ese contexto encontrar la posibilidad, el reconocimiento, las condiciones de implementación, el manejo del tiempo y las prioridades, y avanzar productivamente.

\section{EJEMPLOS DE AFRONTAMIENTO EN EMPRESAS}

\section{Ejemplo 1. Empresa familiar} (conflicto tipo A)

Se trata de una empresa fundada por el padre, de la cual se están haciendo cargo dos hijos. Motivan la consulta las discusiones entre ambos que ocurrían en cualquier momento, en diferentes sectores de la empresa y frente a los 
empleados. Uno ve la situación desde el sector financiero, el otro desde el creativo y la comercialización, y discuten fuertemente por sus diferencias en cualquier momento y contexto. Sin embargo, ambos sectores son fundamentales para el buen funcionamiento de la empresa y necesitan una coordinación adecuada.

La estrategia de afrontamiento diseñada conjuntamente es que los temas sobre los que necesita decidir son importantes y requieren ser dilucidados mediante conversaciones en un contexto que les permita reflexionar y poder tomar decisiones abarcativas que contemplen las implicaciones empresariales de las diferencias. Las discusiones empresariales son tratadas como diferencias fraternas invadiendo la operatoria de la empresa (Fried Schnitman \& Rotenberg, 2009).

La intervención consistió en distinguir espacios para promover mejores conversaciones sobre la empresa, incluyendo las necesidades de las diversas áreas y contextos apropiados para la toma de decisiones: dentro de la empresa se crearía un espacio de reunión para analizar los problemas a decidir diariamente con temario acordado. La reunión podría durar hasta una hora, al comienzo de la jornada laboral; salvo urgencias muy serias que requirieran una definición inmediata, todas las situaciones se tratarían en ese contexto, con agenda y a puertas cerradas. Se estableció una coordinación en la direc- ción de la empresa. Los temas familiares se tratarían fuera de la empresa.

Esta solución se implementó exitosamente y la situación operativa entre ellos mejoró. Diversificaron la empresa familiar y la emprendieron exitosamente. Años después decidieron seguir rumbos diferentes.

Ejemplo 2. Empresa multinacional en transición. Gestión del sí mismo y gestión organizacional (conflicto tipo B que avanza hacia C, se necesita desarrollar recursos para permitir conversaciones productivas)

Se trata de una empresa con filiales en tres continentes, con un sistema de producción distribuido en diferentes países, que diseña máquinas muy costosas según necesidades del cliente. Hasta el momento de la consulta la empresa está conformada por equipos horizontales, cada uno relativamente autónomo y con un alto potencial de innovación. La empresa está creciendo y el diseño productivo implementado hasta ese momento no admite el número de máquinas que necesitará proveer en un futuro cercano. Previendo una crisis en la cual la empresa sufriría enormes pérdidas económicas, el presidente decide reorganizar y centralizar la producción. El ejecutivo consultante -quien había implementado el diseño horizontal con equipos autónomos para el desarrollo de productos- fue designado por el presidente como encargado de llevar adelante la reorganización. Tenía dificultades importantes para avanzar por- 
que consideraba que el incremento de la coordinación involucraba centralizar la empresa nuevamente, abandonar un modelo de equipos altamente creativos e innovadores y exitosos económicamente, para retornar al modelo inicial del que oportunamente había sido muy beneficioso salir. Estaba desorientado, experimentaba angustia, no podía dirimir el marco adecuado para la acción, anticipaba un clima de desconcierto y dificultades para obtener el aval de sus pares a quienes debía ubicar en posiciones subordinadas a la suya, y no podía posicionarse adecuadamente.

La estrategia de afrontamiento diseñada conjuntamente en la consulta consistió en: 1) construir una matriz generativa que permitiera promover conversaciones productivas en lugar de confrontativas; 2) con este propósito, marcar la diferencia entre los tres momentos evolutivos de la empresa y sus implicaciones económico-financieras, relacionales y en el diseño de equipos para compartir la necesidad del cambio; y 3) analizar las ventajas y desventajas de esta transición con sus pares.

Se distinguieron los diferentes momentos de la historia de la organización según los diseños de producción, la rentabilidad, la innovación y los resultados obtenidos, y se caracterizaron de la siguiente manera: un primer momento jerárquico y centralizado; un segundo momento horizontal y descentraliza$d o$; y este tercer momento de coordinación imprescindible para incrementar la eficiencia y sostener la innovación y la rentabilidad, que se define como de "coordinación y co-participación centralizada", diferenciándolo de modelos jerárquicos y horizontales. Cada modelo fue adecuado a un momento del desarrollo de la empresa, que se caracterizó por su agilidad y su posibilidad de transformación.

Dicha distinción proveyó al consultante de una línea argumental y una matriz generativa para validar la transición. Esta intervención puntual le permitió sostener conversaciones productivas con la mayoría de sus pares y sentirse respaldado en su gestión para avanzar la tarea a implementar, si bien en el proceso enfrentó conflictos, desconfianza e incluso amenazas. Otros aspectos que la empresa tuvo que trabajar no formaron parte de esta consulta. Durante el proceso tuvieron lugar situaciones atípicas - desde dificultades de los otros ejecutivos para entender la transición hasta amenazas telefónicasque el ejecutivo pudo afrontar.

\section{Ejemplo 3. Filial de una empresa} multinacional. Afrontamiento colaborativo de una eventual crisis por incumplimiento de metas: Una asamblea de trabajo (conflicto de tipo $B$ en el que se desarrolla una matriz generativa) ${ }^{2}$

Se trata de una clínica de propiedad de una multinacional que opera con un

2 Caso de aplicación del modelo presentado en el seminario "Organizaciones en crisis y crisis en 
modelo estratégico en cuyo marco la relación inversión-beneficio está pautada desde el país de origen. El problema a considerar por el gerente que la administra es que la clínica, a pesar de que presta excelentes servicios y es rentable, no cumple con las utilidades esperadas por la casa matriz: debe mejorar la rentabilidad o corre el riesgo de que cierren la clínica.

El gerente decide innovar el modelo de gestión utilizando una metodología colaborativa y participativa para afrontar la crisis potencial inminente. Convoca a una reunión con todos los profesionales, técnicos y administrativos de la clínica. La reunión comienza con un clima hostil por parte de los convocados, quienes creen que la reunión precede a los despidos y recortes de sueldos. El gerente aclara que no es ese el propósito, sino compartir con ellos el problema para encontrar juntos una solución a las reformas requeridas; de otro modo podrían cerrar la clínica. Las expectativas hostiles previas se transforman, los participantes aceptan la propuesta, consideran las reformas que podrían tener lugar, hacen los recortes y elaboran un plan que les permite llegar a los números que necesitan por caminos que consideran apropiados. Como resultado de

\footnotetext{
organizaciones: Metodologías, destrezas y desarrollo de recursos para la prevención y el manejo de conflictos", en la Carrera de Posgrado de Especialización en Administración de Empresas en Crisis, Facultad de Ciencias Económicas, Universidad de Buenos Aires (2005).
}

la reunión, en ese momento, mantuvieron la clínica funcionando y expresaron que pocas veces en su trabajo profesional se habían sentido tan respetados y reconocidos, aunque se ajustaran costos que los afectaba.

La transformación de la matriz generativa permite pasar de una solución jerárquica a una colaborativa.

Ejemplo 4. Empresa multinacional. Resolución de un problema organizacional, desarrollo de nuevos recursos y matriz generativa en equipo de trabajo (transformación de un conflicto de tipo C) ${ }^{3}$

Esta situación se da en la Gerencia de Administración y Finanzas (GAF) de una empresa multinacional con sede en Chile, la cual ha crecido un $300 \%$ en el último año y medio y proyecta triplicar nuevamente sus ventas en el próximo año y medio. Este crecimiento se explica por el ingreso al mercado masivo de las telecomunicaciones del que hasta hace dos años no participaba. El conflicto se origina en la lentitud de adecuación de la GAF a los nuevos requerimientos en términos de procesos y sistemas para realizar los suministros de equipo y los pagos a proveedores externos. Los proveedores externos (sub-

3 Caso de aplicación del modelo presentado en el curso Gestión de Crisis y Conflictos, en Magíster y Diploma en Psicología de las Organizaciones, Escuela de Psicología, Universidad Adolfo Ibáñez, Chile, 2009. 
contratistas) representan más del 70\% de la fuerza laboral de la compañía. La semana anterior el conflicto original se incrementó, transformándose en una crisis (conflicto tipo $\mathrm{C}$, agudo). Uno de los subcontratistas cayó en insolvencia por no recibir los pagos a tiempo; los trabajadores de ese subcontratista se manifestaron, quemaron neumáticos en el frontis de la empresa y exigieron los sueldos adeudados. Las relaciones entre la GAF y las gerencias de negocios se encontraban en un punto crítico y de quiebre; los otros gerentes señalaban que la GAF constituía un obstáculo para el logro de los objetivos de la organización: "estamos enfocados en lograr nuestro gran objetivo del año... a pesar de la GAF".

De la GAF dependían seis gerentes de unidades: logística, control y presupuesto, facturación, etcétera, que no trabajaban como equipo, pero cada uno había sido exitoso en la gestión técnica de su área. El desafío de la cadena de suministros y el proceso de pago obligó a los gerentes a trabajar entre ellos y con otras áreas de la empresa. La GAF se acercó a nosotros -los consultores- para pedir ayuda porque estaba absolutamente desorientada, reconocía a su gente como "profesionalmente muy competente, pero por alguna razón no logran trabajar en equipo". Las emociones que percibimos en él fueron el miedo y la frustración, principalmente. Al entrevistar a los gerentes del área notamos que la desconfianza, las conver- saciones pendientes, la desesperanza y victimización frente a todo lo que ocurría era transversal a todos ellos. La ausencia de una mirada integradora de las demás áreas fue para nosotros evidente y todo el tiempo en sus conversaciones estuvieron desprestigiando a su jefe y a sus compañeros de equipo. La polarización de las miradas estaba al máximo. Cada gerente de unidad estaba centrado en su objetivo propio, al que consideraba lo único significativo; la eventual falla de otra persona del equipo no le importaba. No existía coordinación de acciones, la capacidad de escucha estaba reducida al mínimo y todos buscaban imponer su propio mundo explicativo.

Nuestra intervención como consultores se orientó a evitar una profundización de la crisis y enfocarlos hacia su afrontamiento. Para ello diseñamos un encuentro fuera de las oficinas, en un lugar agradable, con el propósito de conversar centralmente acerca de aquello que los unía y cuáles eran sus focos comunes, para que pudieran reencontrar y construir un nuevo "nosotros". Iniciamos el encuentro escuchando sus expectativas personales y generando una conversación que los invitó a proponer los cuatro focos que deseaban lograr en ese encuentro. En el proceso fuimos mostrándoles qué diálogos generaban posibilidades de nuevas coordinaciones entre las distintas gerencias y cuáles los separaban. Nuestra segunda acción en ese encuentro fue mostrar el nuevo escenario que se les presentaba como or- 
ganización. La empresa del 2007 -en la cual cada uno había realizado excelentes aportes- hoy requería algo diferente y los desafiaba a trabajar coordinadamente y como equipo. Juntos logramos diseñar nuevos escenarios adecuados a los desafíos de ese momento. Hablamos de las emociones que los habitaban en esos momentos y cuáles habían estado presentes en momentos que percibían como exitosos. La conversación derivó hacia aquello que podía constituir un verdadero desafío de equipo, por el cual cada uno estaría dispuesto a jugársela. Así surgió un gran propósito y una incipiente matriz generativa: "trabajar con excelencia en el área de administración y finanzas, y ser reconocidos así al interior de la organización". A continuación se trasladó ese propósito a acciones cotidianas que pudieran tornarse tangibles. Finalmente, la conversación se enfocó en que el equipo expresara su reconocimiento a cada uno de sus integrantes: qué hace bien, sus talentos y aportes. Hubo importantes transformaciones que marcan un pasaje hacia el incremento de la coordinación, procesos de afrontamiento y la construcción de una identidad grupal y de sí mismos. Trabajaron en la creación de lo común a partir del reconocimiento recíproco de aquello que los une y construyeron una matriz generativa de propósitos, contextos, relaciones e identidad.

En una segunda etapa nuestro objetivo fue participar observando las reuniones de equipos para mostrarles cuá- les de sus diálogos eran generativos y cuáles caían en dinámicas que tendían a polarizarlos. La idea fue que reconocieran los recursos propios y los del equipo, y adquirieran la habilidad de preguntarse generativamente sobre sus circunstancias.

Ejemplo 5. Episodio experiencial de afrontamiento de conflicto y crisis en una familia (conflicto tipo B) ${ }^{4}$

Los participantes en los muchos programas de formación que he realizado en diferentes países reportan una mejora en sus relaciones personales, laborales y sociales, y en su calidad de vida. Este es un hallazgo transversal que presentan en las evaluaciones de seguimiento. ${ }^{5}$

En el ejemplo que sigue -y en otroslos participantes utilizan la perspectiva generativa y sus herramientas para abordar sus propios conflictos.

En esta pareja hubo una propuesta y un compromiso deliberado de construir un campo generativo en la relación (Fried Schnitman, 2000a). Los problemas fueron reformulados en un marco

$4 \quad$ Caso de aplicación del modelo presentado en el curso Gestión de Crisis y Conflictos, en Magíster y Diploma en Psicología de las Organizaciones, Escuela de Psicología, Universidad Adolfo Ibáñez, Chile, 2010.

5 En la actualidad investigo cualitativamente las transformaciones reportadas por los participantes en cursos en diferentes países. También estudio el impacto de la incorporación del modelo en programas organizacionales, comunitarios, rurales, entre otros, que tienen lugar en Cuba. 
apreciativo y generativo, y activamente promovieron posibilidades para sí mismos y su familia. Se transformó la matriz generativa. De una pareja en confrontación y crisis se convirtió en una pareja dispuesta a trabajar colaborativamente en sus diferencias, construyendo consensos, revisando diferencias, enfrentando lo necesario.

\section{MARCo COnCEPTUAL ${ }^{6}$}

- Conflicto. - Las diferencias y los conflictos son inherentes a las relaciones humanas. Las personas tienen diferencias que pueden conducirlas a diversos tipos de proceso en un espectro que va desde la desorientación hasta el encuentro de posibilidades. Nos ocupamos aquí de favorecer los tipos de conversaciones que permiten a las personas encontrar recursos a partir de las diferencias o conflictos.

- Crisis.- En las crisis se desarticula la posibilidad de un funcionamiento implícito. Se pierde la congruencia entre los sistemas explicativos, la experiencia subjetiva, las acciones y el contexto. Se manifiesta por el resquebrajamiento de la dinámica familiar, a veces la ruptura, y una necesidad de adecuación a las nuevas circunstancias que no puede materializarse.

$\overline{6}$ Este marco conceptual se construye sobre la base de las publicaciones de la autora utilizadas como bibliografía para el curso.
- Afrontamiento.- Denominamos afrontamiento de crisis y conflictos al diseño e implementación de procedimientos necesarios para abordar adecuadamente las situaciones específicas de conflicto y crisis, con la aspiración de que resulten efectivos. El afrontamiento es una de las formas de coordinación social que requiere disposición de las personas a trabajar con otros. Implica que las personas puedan vincularse entre sí, escuchar lo que cada uno puede aportar, clarificar contextos y propósitos, poner el foco en los temas a resolver, expresarlos de manera adecuada y construir posibilidades que promuevan recursos para acciones específicas. Abarca la elaboración de supuestos y sistemas explicativos alternativos, la construcción de conversaciones productivas, la colaboración y la coordinación entre los involucrados, con un foco en la resolución del conflicto o crisis en curso.

- Diálogos generativos.- Los diálogos generativos trabajan con una mirada cuidadosa sobre la conversación y el flujo de las conversaciones. Ofrece competencias para sostener rumbos productivos en la conversación, habilidades para generar temas significativos, recuperar recursos, visualizar el futuro, generar involucramiento $\mathrm{y}$ acciones participativas. Pueden integrarse a diversos enfoques y procesos. Trabajan con posibilidades y realidades emergentes para promo- 
ver innovaciones. Requieren una mirada atenta a las experiencias únicas y a los sutiles momentos en que lo novedoso asoma, a la posibilidad de realizar síntesis entre diferentes contribuciones y momentos, de imaginar un futuro. Se focalizan en los episodios capaces de crear nuevos significados y prácticas alternativas (Fried Schnitman, 2000a, 2000b).

Esto implica que es un proceso que requiere diferentes tipos de iniciativas:

- Construcción del dominio del diálogo.- Creación de un contexto adecuado. Facilitación de emociones capaces de sostener el proceso. Exploración de alternativas. Reconocimiento de la singularidad del otro. Promoción del flujo y la diversidad de condiciones de existencia. Creación de matrices generativas de nuevos significados, identidades y relaciones emergentes que permitan construir nuevas perspectivas. Consideración del tiempo y el proceso. Creación de relaciones interpersonales de confianza y conectividad. Recuperación $\mathrm{y}$ construcción de recursos, posibilidades y escenarios novedosos. Trabajo con una mirada cuidadosa sobre el diálogo y sobre el flujo de diálogos. Promoción de rumbos productivos en la conversación, habilidades para generar temas relevantes, construir significados y acciones participativas, recuperar recursos, visualizar el futuro. Trabajo con posibilidades y realidades emergentes para promover innovaciones. Se focaliza en los episodios capaces de crear significados y prácticas alternativas.

- Competencias para el diálogo.Aprender a observar y participar de interacciones dialógicas y conformar equipos colaborativos. Adquirir recursos para participar, escuchar y expresarse, apreciar, identificar y promover innovaciones, reconocer momentos sutiles y novedosos, iniciar movimientos en un diálogo, aprender reflexivamente. Estar atento a la multiplicidad con un foco en el propósito del encuentro y su decurso.

- Saberes.- Identificar tipos de conocimiento emergente. Saber qué, saber cómo, saber cómo decir, saber reconocer un contexto, adecuar(se) y transformar(se), saber acerca de sí en contexto, saber reconocer el campo emocional, saber reconocer el campo social, saber reconocer potencialidades a futuro, saber gestionarse, saber reconocer recursos y buenos desempeños. 


\section{- Innovación}

\begin{tabular}{|c|c|}
\hline Perspectiva & Recursos \\
\hline Dialógica & $\begin{array}{l}\text { - Reconstrucción de posibilidades y oportunidades en } \\
\text { la conversación }\end{array}$ \\
\hline Argumental & - Nuevas formas de elocuencia inclusiva \\
\hline Generativa & $\begin{array}{l}\text { - Reconocer puntos de acceso para nuevas posibili- } \\
\text { dades }\end{array}$ \\
\hline Performativa & - Reconocer interacciones novedosas \\
\hline Narrativa & $\begin{array}{l}\text { - Reordenamiento de la estructura narrativa y del ob- } \\
\text { servador }\end{array}$ \\
\hline Encuadre comunicativo & - Posibilidad de marcos comunes novedosos \\
\hline Centrada en las emociones & $\begin{array}{l}\text { - Registro de las emociones positivas y negativas, y } \\
\text { creación de campos emocionales que favorezcan la } \\
\text { colaboración }\end{array}$ \\
\hline $\begin{array}{l}\text { Centrada en la construcción de } \\
\text { contextos }\end{array}$ & $\begin{array}{l}\text { - Aprender a reconocer o construir contextos que } \\
\text { tengan la intención de promover posibilidades }\end{array}$ \\
\hline
\end{tabular}

Descripción de la situación actual (contexto)

Es una historia familiar y, como todas ellas, en su evolución está cargada de momentos de mucho diálogo y emocionalidad. Son 24 años de matrimonio, con tres preciosas hijas: Juliana (20), Patricia (12) y Manuela (10), y un camino construido desde cero, en el que con el paso de los años se fue creando todo lo que tenemos. Juana, acostumbrada a trabajar desde los 24 años, y yo, un "curiosiador" empedernido, "estar haciendo" es parte de mi ADN. Hace 12 años el nacimiento de Patricia con síndrome de Down hizo que nos uniéramos más como pareja y familia. Ha sido un cami- no de permanentes desafíos y alegrías familiares y personales. Por otra parte, movido por mi curiosidad -conversado y de común acuerdo- decidí independizarme para cambiar de empleado asalariado a consultor de empresas y profesor. Transcurre el tiempo y hace tres años Juana -de común acuerdo- deja de trabajar para dedicarse al desarrollo de Patricia, dado que está evolucionando cognitivamente de forma muy satisfactoria, asistiendo a un colegio normal integrado donde se le realizan las adaptaciones curriculares necesarias. A esto se suma que mi forma de trabajo evoluciona hacia un modelo de teletrabajo en el que una parte importante del tiempo la puedo pasar en una buena oficina 
instalada en casa. Bajo este escenario comienzan a suceder episodios que inician un deterioro importante de nuestra relación. Exigencias mutuas que no son bien canalizadas ni comprendidas por ambos, diálogos centrados en la confrontación donde los juicios y las recriminaciones empiezan a formar parte de la relación, y el alejamiento, un recurso utilizado para dejar que la tormenta amaine.

\section{Observables de la conversación para evaluar la situación}

En este escenario se percibe un conflicto de nivel B: si bien las visiones del mundo son fundamentalmente diferentes, para volverlas coordinables se requiere una transformación de las $m a-$ trices generativas que permita expandir los recursos para aunar esfuerzos y encontrar soluciones. Es necesario expandir o transformar las premisas del sistema de creencias así como las prácticas, instituyendo nuevas matrices generativas. Los recursos comunicacionales y la mediación de significados no son suficientes; se requieren habilidades de otra índole para desarrollar una nueva matriz generativa que permita coordinar significados y acciones con otros. Acotar aquello que es necesario coordinar para sostener acciones conjuntas. Desarrollar una interpretación, significados y un lenguaje comunes que enlacen de manera viable las lógicas y sistemas de creencias que articulan las diferentes visiones del mundo de cada uno de nosotros, y nos permita incluirnos en una visión compartida.

\section{Curso del proceso: Cómo progresa,} cómo participamos, iniciativas que tomamos, evaluación en acción, recursos para la gestión del sí mismo

Basados en el contexto descrito, iniciamos un proceso entre nosotros de diálogo generativo de afrontamiento. La primera pregunta como tema básico es si realmente queremos estar juntos (significado), llegamos a la conclusión de que sí, dado que queremos conservar muchas de las cosas que hemos construido y que nos han unido por siempre. La segunda pregunta dentro del diálogo generativo de afrontamiento es ¿qué debemos cambiar para que mejore la relación? Este era el mayor desafío que enfrentábamos, dado que aquí es donde se debía producir el gran cambio de paradigma de un diálogo de enfrentamiento que cierra posibilidades y que está centrado en el yo, a un diálogo de afrontamiento generativo que abre posibilidades y que debe ser innovador para transformar la actual matriz generativa imperante y centrarse en nosotros.

\section{Las preguntas que emergieron de} este diálogo generativo basado en afrontamiento fueron:

- ¿Cómo creamos un contexto adecuado para que emerjan los diálogos generativos? 
- ¿Qué quiere hacer cada uno de nosotros sin perder la identidad propia y la de familia?

- ¿Cómo podríamos conformar un equipo colaborativo centrado en lo que nos une?

- ¿Cómo podemos vitalizar nuestro futuro?

- ¿Cómo podemos generar emociones positivas en cada una de nuestras conversaciones generativas?

\section{Basados en estas preguntas}

fundamentales hemos ido reconstruyendo nuestra matriz generativa donde las acciones han sido:

- Reconocer la importancia que tiene el trabajo que realiza Juana con Patricia y nuestras otras dos hijas.

- Incrementar nuestro espacio común como pareja, viajar juntos cuando las circunstancias lo permiten para compartir y estar juntos en viajes largos que debo realizar por mi trabajo.

- Arrendar oficina, para que el tiempo en la casa no se mezcle con el laboral.

- Disponer de los fines de semana como una instancia familiar y de pareja.

\section{Los resultados:}

Una relación más fluida que pasó de la confrontación al afrontamiento. Diálo- gos generativos centrados en abrir posibilidades innovadoras desde el "hacer juntos". Surgimiento de emociones positivas que abren posibilidades y energizan la relación.

\section{Ejemplo 6. Crisis en una empresa} familiar. Desarrollo de una matriz generativa y recursos de coordinación y gestión (conflicto tipo $\mathrm{C}^{7}$

Se trata de una empresa familiar del rubro de pastelería. La consulta la realiza uno de los socios. Esa noche los socios tendrían una reunión para decidir el futuro de la sociedad. La sociedad está integrada por dos parejas de primos, cada uno de los cuales tiene una especialidad y es muy talentoso: una pastelera muy creativa, un abogado, una especialista en márketing y un asesor de negocios. La pastelería se inició un año atrás, trabaja muy bien pero no consigue equilibrarse financieramente debido a que no logran coordinar aspectos creativos, comerciales y administrativos, generando muchas diferencias entre las dos parejas de socios. La pastelería es excelente y muy creativa e incorpora novedades permanentemente. Si bien la empresa no alcanza su punto de equilibrio las ventas son muy buenas y se

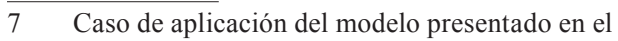
curso Gestión de Crisis y Conflictos, en Magíster y Diploma en Psicología de las Organizaciones, Escuela de Psicología, Universidad Adolfo Ibáñez, Chile, 2009. 
instaló bien en el mercado. Los socios tienen ideas diferentes respecto de la coordinación y desarrollo del negocio; no pueden sostener un curso de acción $\mathrm{y}$, cuando deciden alguno, no lo mantienen. La pastelera genera nuevos productos permanentemente y diversifica la oferta, el asesor de negocios opina que deberían limitar el número de productos hasta alcanzar el equilibrio financiero. No pueden coordinar una estrategia conjunta. Incorporaron un gerente administrativo para mejorar la coordinación pero no funcionó. El consultante dice que acordó con su pareja ceder el paquete accionario al marido de su prima. Esta empresa surgió de la buena relación entre los cuatro participantes, pero esta se deteriora cada día más. El consultante plantea que para preservar la relación está dispuesto a ceder su parte pero ve que si la empresa sigue en ese rumbo, aun con su salida, no será viable. En el relato se presenta a sí mismo en una situación paradojal que no reconoce como tal: está fuertemente involucrado pero planteando que se va, al mismo tiempo no quiere administrar la empresa (tiene otros negocios) pero la empresa le importa y se involucra. Comenzamos a trabajar planteándole la importancia de que decida si quiere quedarse o irse, no puede estar adentro y afuera al mismo tiempo porque implica caminos diferentes. Decide quedarse. Le pregunté si en la sociedad habían tenido experiencias exitosas de diseño, coordinación e implementación de un plan. Recuerda que sí y las enumera. Le pedí detalles acerca de qué les permitió coordinar y cómo lo hicieron, y qué había sucedido en las que no habían logrado coordinar. Sobre la base de esta información le solicité que trabajara en una apertura para la reunión de esa noche, la consigna fue que recuperara los aportes de cada uno de los socios a las experiencias que habían funcionado bien, y a la sociedad misma. Con este material él construyó una nueva matriz generativa con la que encuadró la reunión de la noche; dicha matriz incluía los recursos que cada socio ofrecía a la empresa y los aportes que había realizado. Esta matriz destacó la importancia de la contribución específica de cada uno, la buena combinación de vínculos y talentos, y rescató para la memoria del grupo las experiencias exitosas en las que habían coordinado, los aportes, y cuánto significaba para cada uno tanto la relación como la empresa. En este marco pudieron encaminar productivamente la conversación, ordenaron prioridades y tiempos, y tomaron compromisos que pudieran sostener.

Tres meses después el consultante reporta que cada vez que van a tener una reunión de socios vuelve a mirar cuál es la matriz generativa que necesitan para seguir avanzando. 
Reflexión del consultante:

Frente a cualquier situación actual, ya sea de conflictos propios o ajenos (en mis consultorías), a partir de participar en el seminario tengo presente la noción "diálogos generativos" y los miro desde ese prisma. Busco descubrir de qué manera podríamos construir un diálogo generativo. Se me ordenaron la perspectiva y los criterios para mirar los conflictos, al punto de darme cuenta de que cuando estamos frente a una crisis (conflicto tipo C), es necesaria la intervención de un tercero, entonces, por ejemplo, si soy el consultor y estoy en el proceso de empoderar a un equipo, lo que hago es intervenir promoviendo nuevos recursos, ya que en ese momento el empoderar no es lo que necesitan. Siento que mi manera de conversar se transformó, y hoy es más generativa. Busco principalmente reconocer en los otros sus potencialidades y sus recursos actuales y de experiencias anteriores, incluso en mí, muchísimo más que antes. De hecho yo he tenido el juicio, y aún me pasa, que me centro mucho en las brechas y lo que hay que mejorar, más que en lo que sabemos y somos buenos para hacer, es decir, la contribución de cada parte y desde el curso hasta ahora, y voy a seguir aprendiendo y teniendo conciencia de ello, busco que cada uno de los participantes nos centremos en lo que tenemos, sabemos $\mathrm{y}$ hemos hecho bien.

\section{REFERENCIAS}

Berger, P. L., \& Luckmann, T. (1966). The social construction of reality. Nueva York: Doubleday.

Bernstein, R. J. (1985). Beyond objectivism and relativism. Filadelfia: University of Pennsylvania Press.

Drucker, F. P. (1999). Management Challenges for the 21st Century. Nueva York: HarperBusiness.

Fried Schnitman, D. (1989). Paradigma y crisis familiar. Psicoterapia y $\mathrm{Fa}$ milia, 2 (2), 16-24.

Fried Schnitman, D. (2005). Afrontamiento de crisis y conflictos: Una perspectiva generativa. Sistemas Familiares, 21 (1-2), 98-118.

Fried Schnitman, D. (2008a). Diálogos generativos. En Rodríguez Fernández, G. (Comp.). Diálogos apreciativos: El socioconstruccionismo en acción (pp. 17-48). País VascoMadrid: Instituto Internacional de Sociología Jurídica Oñati-Editorial Dykinson.

Fried Schnitman, D. (2008b). Diálogos generativos y su aplicación a organizaciones. Psicología Organizacional Humana, 1 (1), 101-115.

Fried Schnitman, D. (2009). Diálogos generativos e indagación apreciativa: Perspectivas y herramientas para el diálogo en/entre organizaciones. 
En Schmukler, B. (Coord.). Coconstruyendo el espacio de la cooperación internacional: Evidencias de la evolución en el vínculo academiaOSC (pp. 49-95). México D. F.: Instituto de Investigaciones José María Luis Mora.

Fried Schnitman, D. (2010a). Procesos generativos en el diálogo: Complejidad, emergencia y auto-organización. Pensando la Complejidad, VIII, enero-junio, 49-60.

Fried Schnitman, D. (2010b). Perspectiva generativa en la gestión de conflictos sociales. Revista de Estudios Sociales, 36, 51-63.

Fried Schnitman, D. (2010c). Conversaciones, diálogos y posibilidades productivas en la mediación prejudicial. Ponencia presentada en el Primer Congreso de Mediación Prejudicial y Judicial "Reconocimiento y Proyección de la Práctica". Unión de Mediadores Prejudiciales. Buenos Aires, 4-6 de noviembre.

Fried Schnitman, D., \& Rotenberg, E. (2009). Negócios e famílias: Promo- vendo recursos. Pensando Famílias, 13 (2), 33-58.

Fried Schnitman, D., \& Schnitman, J. (2000a). La resolución alternativa de conflictos: Un enfoque generativo. En Fried Schnitman, D. (Comp.). Nuevos paradigmas en la resolución de conflictos. Perspectivas y prácticas (pp. 133-158). Buenos AiresBarcelona-México-Santiago-Montevideo: Granica.

Fried Schnitman, D., \& Schnitman, J. (2000b). Contextos, instrumentos y estrategias generativas. En Fried Schnitman, D. \& Schnitman, J. (Comps.). Resolución de conflictos. Nuevos diseños, nuevos contextos (pp. 331-362). Buenos Aires-Barcelona-México-Santiago-Montevideo: Granica.

Pearce, W. B., \& Littlejohn, S. W. (1997). Moral conflict. When social worlds collide. Thousand OaksLondres-Nueva Delhi: Sage Publications.

Prigogine, I., \& Stengers, I. (1979). La nouvelle alliance: Métamorphose de la science. París: Editions Gallimard. 